\title{
Digital Initiatives, Infrastructures and Data Ecosystems in the Maritime Sector
}

\author{
Socrates Theodossiou, Navneet Singh Rainu, \\ Tototheo Maritime, Cyprus
}

\section{Synopsis:}

Efficiency, performance and monitoring of vessels becomes of paramount importance around the globe. Assets security, vessels efficiency, new directives and legislation with regard to emissions quality and many others, urge the global maritime industry to take the right initiatives and make the appropriate investments to develop data ecosystems, that over time, if used intelligently, coherently and consistently, will allow owners and managers to reap tangible benefits such as, among others, significant cost savings, better vessel management and longer vessel life span.

As of today, most shipowners and related stakeholders face huge challenges when it comes to data collection, processing, streaming, sharing and storage. Relevant data, if any, is isolated in distinct silos, in spurious and inconsistent formats with little or non-existent interconnectivity between such silos or storage mechanisms. In effect, to face the new challenging landscape, a fresh mindset and an open-minded approach is required.

The paper uses data and relevant building blocks, related to vessel performance, assets tracking, route planning, engine monitoring, fuel consumptions, emissions quality, vessels tracking, performance alarms and notifications; that is a wide variety of data modules and reporting tools, that eventually serve pure reporting, real time monitoring and visualization objectives; but also some additional, more powerful modules being used for analytics and strategic decision making. Such modules can leverage on historical data being captured over prolonged time periods, in the various interrelated data sources and by the relevant data collectors and, if deployed effectively, to construct supervised, unsupervised or even semisupervised machine learning models. Eventually, such models will enable the various stakeholders in this domain, to achieve successful assignments related to predictions, regressions, classification and clustering. In effect, apart from pure vessel geolocation tracking capabilities, the above modules and tools will allow any shipowner to log-in and see how, a specific vessel under consideration does, in terms of performance and efficiency, in specific weather, geological and regional conditions. In addition to that, more advanced modules, for instance, might warn ship owners about the benefits of a potential hull maintenance or cleaning, give insights on engine efficiency and recommend actions or even provide indications or predictions of future likely delays in reaching at the port of destination.

Among other things, this data collection and storage, in such a digitalization platform (will) allows the ongoing building-up of insights, knowledge and technical expertise associated to (optimized) vessels performance and all related functionalities as stated elsewhere. As the variety, veracity, volume and quality of the collected data, across the board, will be gradually enriched, enhanced and improved over time, allowing relevant stakeholders to gain real benefits, such as potentially reduced costs triggered by better and proactive vessels management, from such initiatives that might look and sound meaningless at the very beginning.

The Paper builds upon the empirical evidence and relevant data associated to Tototheo Maritime's, Digital Control Room and its associated Maritime Digitalization Platform that do provide, not only a state-of the art platform that facilitates visualization and snap-shot reporting functionalities but also modules upon which machine learning and advanced analytics can be deployed.

Keywords: Digitization, Ecosystem, Maritime, Transportation, Analytics, Vessel Performance.

\section{Introduction}

What does it take to optimize fuel consumption? Theoretically, it is simple. The fuel should be consumed efficiently onboard, consumption trends analyzed, and conclusive decisions should be taken to maintain optimal state, if not improve it. In real life application however, these processes are handled at different points in time, by different persons or departments and using different tools. 
So, what can we do to monitor our fleets' consumption and hope to improve the voyage earnings? Optimization Literature show that there is an urge in the industry to come up with ideas to reduce fuel consumption to improve voyage earnings and conserve environment.

Averagely $60-80 \%$ of the voyage costs are towards bunkers and with the fuel prices as volatile as they are in recent times, the need to optimize fuel consumption has become almost a requirement for those wishing to remain competitive. To make matters more complex we also need to deal with the EU MRV (European Union Monitoring, reporting and verification) and IMO DCS (International Maritime Organization - data collection system) regulations, which means an additional cost for someone monitoring, reporting and validating the emissions as a service.

When talking about Voyage Optimization there are several questions most commonly being asked. The first one can be a simple one - where are my assets located at any time? We start with seeing the ships on a chart - a visual representation helps. Next, one might think about the routing to the destination port. Until recently, the shortest and safest route would be the obvious choice for even the most experienced master. Today, we see masters adding the "efficiency" factor also in their passage planning. The shortest route may not be the optimal one and any sailor knows the general passages to capitalize on the ocean currents.

While planning passages, navigating officers find it useful to have another experienced colleague, onboard or ashore, to discuss thoughts and bounce ideas off. Not necessarily to draw the shortest distance between two points but to get information on the weather, traffic, expected speed to arrive at the communicated time of arrival. In reality however, the ships are running on the leanest crew compliment in history.

It takes an organization who appreciates the value of the environment and truly considers it part of their responsibilities as a global operator, to protect it, to make the mindset shift towards real optimization. Only through this realization can an organization explain their investment in such practices. Answer lies in having "routing buddies" or subscription to routing services by 3rd parties. The impact of such investments on the bottom line may be delayed in being evident overall and a deep understanding and motivation is required to recognize the benefits. Undoubtedly, beneficial results become obvious through voyage earnings which becomes an acid test to see if the optimization steps taken are working. Believe it or not, even under an experienced master's command, many passage plans have scope for tweaking and times huge savings can be squeezed out of seemingly straight forward and short voyages depending on the season and passion with which the original passage was planned. The use of ECDIS really puts this issue under a debate. ECDIS through its significant potential as a navigation tool, enables the user to save routes so that they can be reused at later occasions when taking the same voyage. However, it is easy to appreciate that just by considering the change in season, the saved route can be vastly inefficient due to different currents, weather conditions and other factors.

Other area to reduce energy wastage is hull and machinery performance optimization. There are various forms of noon reports being produced, every day, per ship. Masters and chief engineers tirelessly filling up reports hoping that all this data over the years is being transformed into added value for their organization and vessel. Truth be told, it is neither managed correctly nor capitalized upon sufficiently for any real business intelligence to be generated from it.

It is not easy for the technical or marine superintendents and vessel operators to monitor their vessel. The digitization of several processes and services, has indeed provided organizations with a lot of data. One might say at times, too much data. Consider this, while a superintendent or operator keeps getting more and more information, they also get more and more vessels added to their profile. There is no time to optimize current voyages while they are gathering information to secure the next one or handling claims from the previous one. To maximize voyage earnings, their main job should be to make the current voyage more commercially profitable and keep OPEX (operational expenditure) low. Let's also not forget that this needs to be happening in almost real time in order to reap actual benefits.

What can be done to assist them? The answer lies in supplementing them with the right tools and a dedicated team whose sole purpose is to optimize voyages, offering advice to the superintendents and if required, have a "sailor-to-sailor" conversation with the captain or the chief engineer on board. These are the considerations which triggered the development of the Digital Control Room by Tototheo Maritime. Our DCR is easily transformed into "Your" own bespoke control room.

In addition to the above factors to tackle them when debating voyage optimization, the role of ports must also be considered. After all, the freight earning element is the cargo and we need ports not only to load and offload cargo, but also to participate in the optimization scheme. Tototheo, has understood that real optimization can only be achieved when looking at the various sections of a vessel's voyage as a real ecosystem where all actors and processes are interconnected and interact with each other on a continuous basis. How else can one face the speed and complexity with which business is carried out today? What is the answer here? Part of the answer lies in collaborations. 
Let's consider the scenario where a superintendent has applied all the expertise and has closely worked with the master onboard, they have both utilized all the smart technology at their disposal and the vessel has executed a truly optimized voyage, reaching port only to be advised that the port is shut due to congestion, weather or that the cargo is not ready. It is a common occurrence. The master then has the option to drop anchor or start sailing in circles to kill time because the ship's staff does not want to wait in maneuvering mode for an uncertain amount of time. Yes, sailing in circles does happen even today and there are reasons for it. None convincing.

Let's take one further step forward and consider a situation where the port informs the master of the current status and average times for the pilot to board, for the hose to connect and for the cargo to start. Masters and ship operators will be better enabled to take informed decisions on the port call. Maybe the vessel could have slowed down five days earlier by half a knot or two to reach port just in time with optimal RPM, resulting in higher voyage earnings.

This not a vision of the distant future, but rather a real opportunity that can be applied to your organization and your assets.

\section{Aspect of vessel optimisation}

\subsection{Advance analytics and Predictive model:}

Analysing hull and machinery performance is a challenge because it is an aggregation of many factors. To make it simpler to your mind's eye, it may help to split the factors. If you see the figure above, we have the aggregate effect of engine, propeller and hull conditions giving us a certain speed. Depending on the engine condition, a certain amount of torque is produced. This torque needs to be transferred as thrust to overcome the resistance from hull to produce the necessary speed.

First step after understanding this is data collection and sanity check to make sure that it is reliable. Frequency of data has an impact on the modelling as shown below.

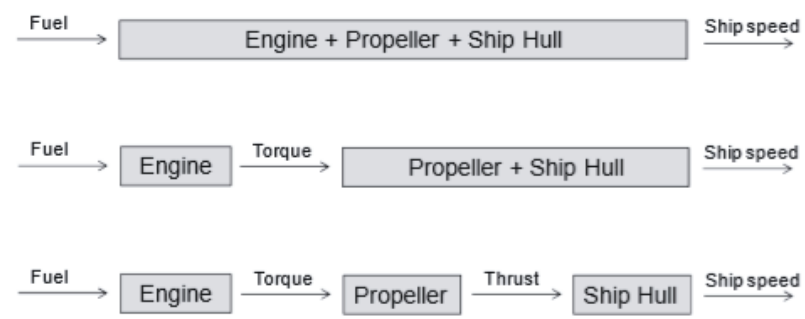

Figure 1: Breakdown of fuel consumption

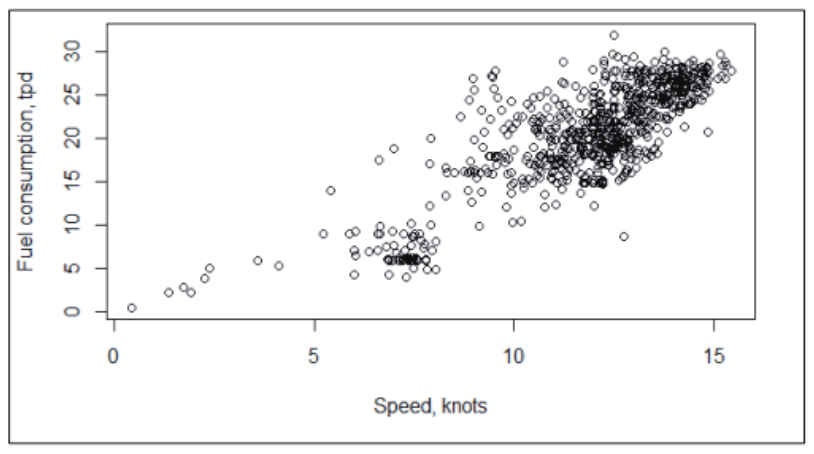

Figure 2: Scatter with noon reports.

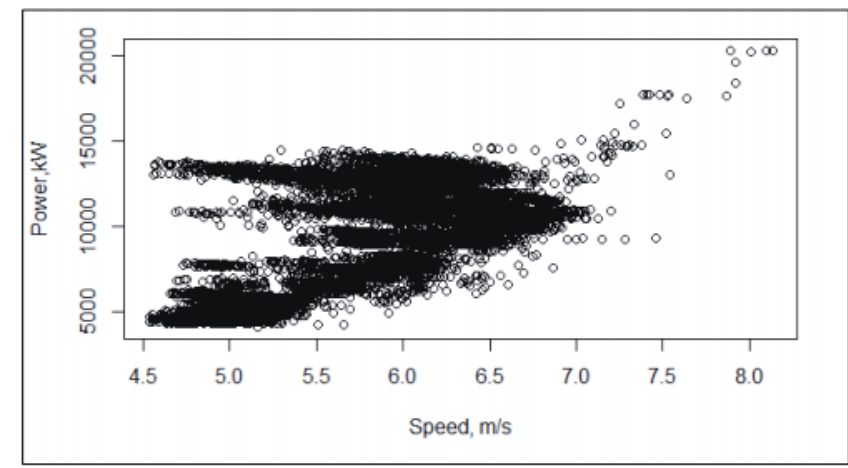

Figure 3: Scatter with continuous live monitoring

\subsection{Creating value from Ship data}

A core element to delivering high-quality, valuable data, is checking, and checking the data again. When data is passed onto further parties, the quality of the data is often questioned. "Accuracy will vary, and you need to know that quality checks are being done to ensure what data you have is accurate," she explains. Data also needs to be validated and an understanding of the physical boundary conditions should be sought. This is about more than just checking data coming in and going out but includes understanding the physical set up of it, which is "absolutely important to pass the data on to the next party."

Ms Lim explains that each step of validation is paramount, and while it may be tedious because it needs to be customized to each vessel, it is a vital step to make sure the next party can see exactly where and how the data has been validated as they will not be able to do this themselves. This also helps when putting data into context. Ms Lim explains that data are just data and can look like a lot of numbers without much context, but if they are broken down to see what they actually represent in the wider context, it becomes clear how much time or energy is spent on each task. In doing so, more value is created from that data. Validating the data for quality is also central to integration with data from the port and the database from the market. "This allows proper sense to come out of it. Otherwise, garbage in = garage out." 
Different routes to creating true value from ship data according to Serena Lim. Ms Lim believes that the current bottleneck is on how the industry can make proper and effective use of data that contain too much information. She believes that the most important aspects are to ensure that systems are carefully developed and optimized for ship management while finding suitable partnerships it is a must-do. "If you stand alone then it can be difficult to provide a holistic

approach, but by finding reliable, active partners, then it is possible."

Quoted from Dr Lim's presentation on Data management.

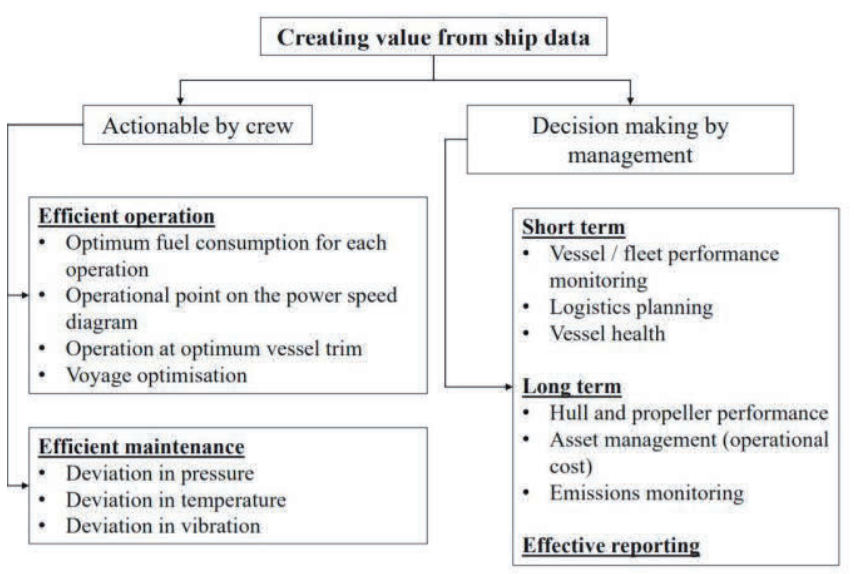

Figure 4: Process flow of value of ship data

\section{$2.3 \mathrm{KPI}$ (Key performance indicators) to optimize fuel:}

\subsubsection{Specific fuel oil consumption (SFOC)}

Specific fuel oil consumption is the measure of the mass of fuel consumed per unit time to produce per KW. The marine engine efficiency is usually determined using the SFOC.

In order to achieve accuracy, the fuel consumption and power developed is always measured over a suitable time period on a good weather. The formula used for calculating SFOC is:

\section{SFOC $(\mathrm{g} / \mathrm{kwh})=$ Mass of fuel consumed per hour $/$ Power developed in KW}

- The readings of the flow meter to the main engine should be noted over the specified time interval say 1 hour. With the difference in readings, the volume of fuel consumed is obtained. It can also be measured by noting down the HFO (Heavy Fuel Oil) service tank reading provided the oil is being supplied only to the main engine.
- The mass of the observed volume of fuel consumed can be determined by following the above-said bunker calculation procedure.

- The horsepower can be measured using dynamometer if fitted on the shaft of the engine which will indicate the BHP in the digital indicator. If not, the horsepower can also be calculated using engine rpm and average pump fuel index with the aid of engine characteristic curve of various sea trials which is supplied by the manufacturer. However, the calorific value of the fuel used for sea trialmay differ and hence compensation factor has to be determined to obtain the accuracy in calculation.

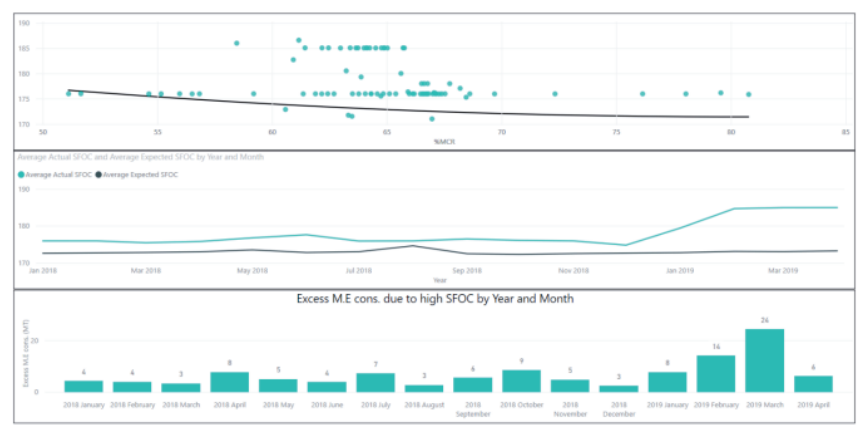

Figure 5: Inefficiencies due to main engine performance

\subsubsection{Hull performance:}

is to measure how much shaft power was applied to attain a certain speed through water. Speed through water is used to negate the effect of current in the measurement. It depends on the resistance build up on the hull due to biofouling or paint degradation. Ideally, once the speed loss crosses the hull degradation threshold, hull cleaning should be considered.

\subsubsection{Propeller performance:}

Slip is considered as the difference between the speed of the engine and actual speed of the ship. It is always calculated in percentage. Positive slip is influenced by various reasons such as fouled bottom or hull part which resists the movement of the ship, environmental factors such as water current and wind against the ship direction. The slip may be negative if the ship speed is influenced by following sea or wind. Engine slip is calculated daily onboard the vessel and recorded in the logbook.

Engine distance - Observed distance

Percentage of Slip $=--------------X$ $100 \%$ 
- $\quad$ The actual distance (nautical mile) covered by the ship from noon to noon is measured using ship's log.

- The total revolutions of the propeller from noon to noon is obtained using revolution counter. The engine distance can be calculated using the pitch of the propeller provided by the manufacturer.

- $\quad$ Engine distance in nautical mile $=($ Pitch $x$ revolutions per day)

Obtaining the accuracy of value in all the above calculations are always challenging onboard as the parameters recorded are more sensitive to dynamic conditions of the ship and depends on various environmental factors.

\subsection{Comparison with a class of vessels}

If a fleet consists of sister class vessels, it is prudent to benchmark them against a baseline created for the class to see how each ship is performing. Baseline created from sister ships are more reliable than shop tests. Baseline created from real world observations is the most accurate that we have.

Data management is a challenge for any shipping entity. The flow of data needs to be thought of. From data collection means to sanity checking to representation. Below is a sample of representation of vessels of same class and a baseline is designed for the ships of the class.

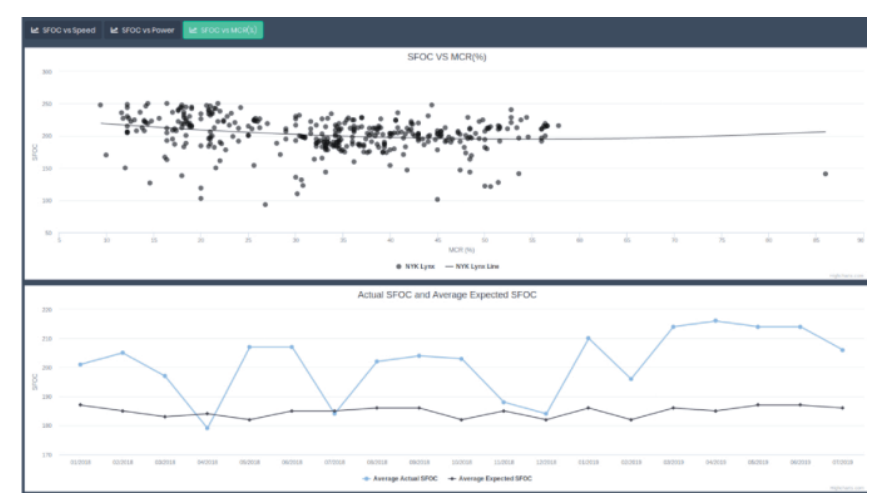

Figure 6: Inefficiencies using SFOC scatter

\subsection{Speed Loss:}

This is an interesting topic because it equips the observer to take actionable decisions based on degradation of performance. How does one set benchmarks to come to this conclusion? Consider the following statement: If the condition of the hull is same as the reference model than the Speed Percentage is $100 \%$. A loss of $0.5 \%$ in Speed Percentage is expected every year after birth. i.e. $2.5 \%$ for a five-year cycle, which is the duration between 2 dry docks. So, a vessel just out of dock and having a SP of $100 \%$ is expected to be around $97.5 \%$ at the end of its first 5 years. After dry dock SP is reset at $99.7 \%$. i.e. $0.3 \%$ is set to compensate for the hull treatment for every docking. 1\% loss of Speed \% results in $3.5 \%$ of extra power on the ME.

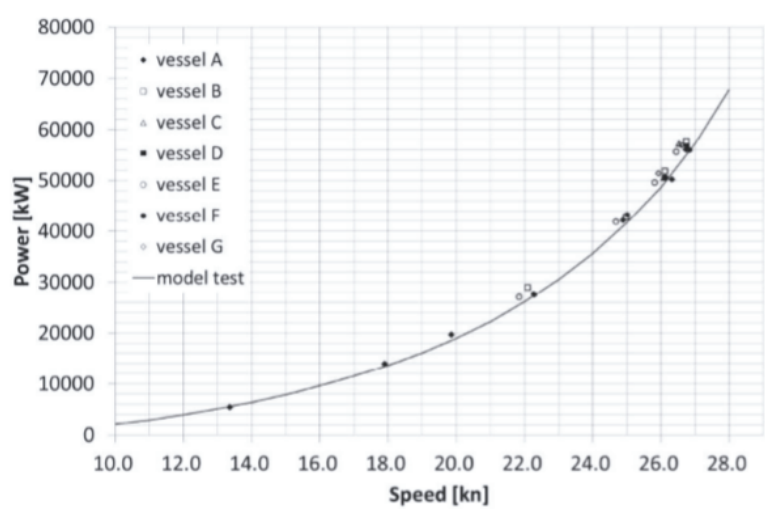

Figure 7: Power vs speed relation

\subsection{Predictive model:}

Up until recently, optimization solutions only looked at historical data, processed and analyzed them to act based on the trend lines. But how about predicting the consumption and required Power or RPM on the route to attain a certain speed? Speed is the determinant be it for ETA, Laycan or Charter party compliance. It is not as straightforward as extrapolating the trend lines from hindcast and this is because of the weather dynamics. To get the results as close as possible to the actual effects, we need to determine the vectors magnitude and direction for wind, wave, swell and current and then use machine learning in the big data available - both hindcast and forecast.

To summarize the factors affecting the energy consumption is represented below:

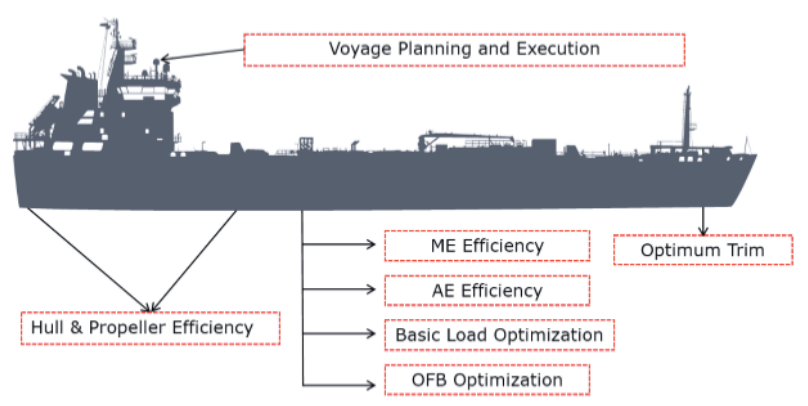

Figure 8: Areas for optimization

\subsection{Trim Optimization:}

Most ships are designed to carry a certain amount of cargo at a designated speed, consuming a certain amount of fuel under a specified trim condition. Loaded or ballast, trim has a significant influence on the resistance of the ship through water. Therefore, optimizing the trim can deliver significant savings. 
For any given draft and speed, there is a trim condition that gives minimum resistance. Therefore, the vessel optimum trim is a function of draft and speed. A ship's optimum trim may be established as part of routine operations or through tank testing or use of computational methods. Nowadays, Computational Fluid Dynamics (CFD) methods are extensively used to estimate optimal trim settings for energy efficiency. However, these may require information from ship model tests and /or full-scale measurements.

Computational Fluid Dynamics (CFD) is a branch of fluid mechanics that uses numerical methods and software systems to solve and analyze problems that involve fluid flows including liquids and gases. In shipping, it is widely used for estimating ship resistances and improves ship design via reduction of number of trial and errors experimentations such as ship's model tank testing. CFD methods involve significant level of numerical calculations; thus, high computer powers that is readily available. On-going research and development have yielded much more accurate estimation of a ship's resistance and powering using this technique; including its use for establishing a ship's optimal trim.

Operationally, design or safety factors may preclude the full use of trim optimization. The possibility of trimming a ship should be seen in relation to stability, maneuverability and other safety and operational aspects. It is the master or chief officer of the vessel that will ultimately ensure all situations are considered. To ensure best practice, the concern of masters with loading and ballasting aspects needs to be considered.

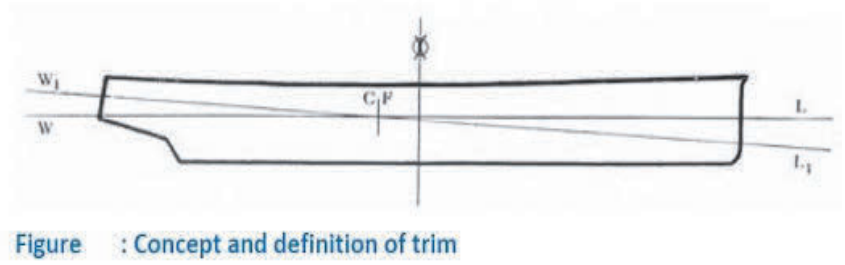

\subsection{Economic benefits:}

Trim optimization has significant economic benefits in terms of fuel savings. These economic benefits will vary from one ship size and type to others. It should be emphasized that even small trim changes can have a large impact on vessel performance. A $2 \%$ to $4 \%$ reduction potential in fuel consumption is generally referred to in most literatures. However, depending on ship type and operation draft, this number may be higher or lower. Therefore, for energy saving, all possible measures should be tried to help with ensuring that this potential is realized. Sailing just 5-10 centimeters off optimal trim might cause ships to operate at higher fuel consumption levels than normal.

There is a bulk of evidences on significant fuel saving potentials due to trim. Ship resistance is altered due to the trim of a vessel through viscous resistance which is a function of the wetted hull surface area. When trim changes, wetted surface area and thereby hull resistance will be affected. By definition, if resistance increases, fuel consumption and emissions also increase.

Thus, to reduce fuel consumption and emissions, trim needs to be optimized before and during a ship's voyage with a proper loading plan of the cargo or use of ballast water in order to achieve a hydrostatic position that consumes the least propulsion power.

Ships normally record their trim before the voyage by directly reading the draft marks. So, considering that the weight distributions on the ship allow trim adjustment, finding the appropriate and optimal floating position before voyage becomes possible (this is referred to as "static trim" when ship is not sailing). However, knowing the exact trim and draught during a ship's voyage is important. The trim under operational condition is normally referred to as "dynamic trim" and is different from "static trim" due to the impact of ship motion. Its measurement requires real-time readings through sensors and relevant on-board data systems.

\section{Conclusion:}

Digitalisation in the shipping industry is a necessity. The industry runs slower to adapt changes than other because of the sheer variety, location, interests and role of the stakeholders that a regulation is required for an effective change management. The other consideration is the cost of research and development that individual companies cannot bear or choose not to plan for in their budget.

Services companies are currently pioneering newer ways as they can specialise in a certain area with the right skillset and commercialise it. This is where they risk investing in a business model to be recognised as an evolutionary idea to help the industry.

\section{References:}

Ship Operational Efficiency: Performance Models and Uncertainty Analysis by Lucy Gemma Aldous, a thesis submitted in fulfilment of the requirements for the degree of Doctor of Philosophy, University College London 2015

Dr Serena Lim's presentation on Data management 